\title{
Antibacterial Resistance of Community-Acquired Respiratory Tract Pathogens Recovered from Patients in Latin America: Results from the PROTEKT Surveillance Study (1999-2000)
}

\author{
C. Mendes, M. E. Marin, F. Quiñones, \\ J. Sifuentes-Osornio, C. Cuilty Siller, \\ M. Castanheira, C. M. Zoccoli,H. López, \\ A. Súcari, F. Rossi, G. Barriga Angulo, \\ A.J.A. Segura, C. Starling, I. Mimica \\ and D. Felmingham
}

\author{
Fleury Centre of Diagnostic Medicine; Laboratory of \\ Microbiology (UFSP); Clinical Hospital of São Paulo \\ University; Santa Casa de Misericórdia, São Paulo, SP; \\ Santa Luzia Laboratory, Florianópolis, SC; Exame Laboratory, \\ Brasília, DF; São Francisco de Assis Hospital, Belo Horizonte, \\ MG, Brazil; Foundation of the Centre of Infectious Diseases; \\ Centre of Infectious Diseases, Buenos Aires, Argentina; \\ National Institute of Respiratory Diseases; Salvador Zubiran \\ National Institute of Nutrition and Medical Sciences, Infectious \\ Diseases Hospital of La Raza IMSS Centre Medical, Mexico, \\ Mexico; GR Micro Ltd, London, UK
}

\begin{abstract}
PROTEKT (Prospective Resistant Organism Tracking and Epidemiology for the Ketolide Telithromycin) is a global surveillance study established in 1999 to monitor antibacterial resistance of respiratory tract organisms. Thirteen centers from Argentina, Brazil and Mexico participated during 1999-2000; they collected 1,806 isolates (Streptococcus pneumoniae 518, Haemophilus influenzae 520, Moraxella catarrhalis 140, Staphylococcus aureus 351, S. pyogenes 277). Overall, $218(42.1 \%)$ of the $S$. pneumoniae isolates had reduced susceptibility to penicillin, $79(15.3 \%)$ were penicillin-resistant and $79(15.3 \%)$ were erythromycin-resistant. Mexico had the highest prevalence of penicillin $\mathbf{( 7 6 . 5 \% )}$ ) and erythromycin $(\mathbf{3 1 . 2 \%})$ resistance. Of 77 erythromycinresistant $S$. pneumoniae tested for resistance genotype, 43 possessed mef(A), 33 possessed $\operatorname{erm}(B)$ and 1 possessed both $\operatorname{erm}(B)$ and mef(A) mechanism. All $S$. pneumoniae isolates were fully susceptible to telithromycin, linezolid, teicoplanin and vancomycin. Among $\mathrm{H}$. influenzae isolates, $88(16.9 \%)$ produced $\beta$-lactamase, ranging from $11 \%$ (Brazil) to $24.5 \%$ (Mexico). Among M. catarrhalis isolates, $138(98.6 \%)$ produced $\beta$-lactamase. Twenty-four $(8.7 \%)$ of the $S$. pyogenes isolates were erythromycin-resistant; resistance being attributable to $\mathrm{mef} A(\mathrm{n}=18)$, ermTR $(n=5)$ and ermB $(n=1)$. All $H$. influenzae, $M$. catarrhalis and $S$. pyogenes were fully susceptible to telithromycin. Methicillin resistance was found in $26.5 \%$ of the $S$. aureus isolates (Argentina 15\%; Mexico 20\%; Brazil 31.3\%). Telithromycin was effective against $97.7 \%$ of methicillin-susceptible isolates. PROTEKT confirms that antibacterial resistance is an emerging problem in Latin America. The previously reported high levels of pneumococcal resistance to the $\beta$-lactam and macrolides were exceeded. New agents that do not induce resistance or that exert low selective pressure, e.g. telithromycin, are essential to safeguard future antibacterial efficacy. Key Words: PROTEKT, Latin America, resistance, respiratory tract infections, Streptococcus pneumoniae, Haemophilus influenzae, Moraxella catarrhalis, Streptococcus pyogenes, Staphylococcus aureus.
\end{abstract}

Received on 20 November 2002; revised 24 January 2003. Address for correspondence: Dr. Caio Mendes. Fleury Centro de Medicina Diagnóstica. Av. General Waldomiro de Lima, 508, São Paulo, SP, Zip Code: 04344-070, Brazil. Phone.: 0055 11 5014-7647. Fax : 005511 5014-7601.

E-mail: caio.mendes@fleury.com.br

The Brazilian Journal of Infectious Diseases 2003;7(1):44-61 (C) 2003 by The Brazilian Journal of Infectious Diseases and Contexto Publishing. All rights reserved.
The prevalence of antibacterial resistance among pathogens commonly involved in community-acquired respiratory tract infections (RTIs), including Streptococcus pneumoniae, Haemophilus influenzae and Moraxella catarrhalis, represents a global problem and appears to be increasing in many countries in Latin America. In particular, $\beta$-lactam and macrolide resistance among $S$. pneumoniae has reached high 
levels in several Latin American countries, including Argentina, Brazil and Mexico. For example, in their analysis of 264 Latin American pneumococcal isolates as part of the SENTRY programme, Odland et al. [1] found that $9.9 \%$ were fully resistant to penicillin. Similarly, of the 1,100 S. pneumoniae isolates from seven Latin American and Caribbean countries surveyed by the LASER study group in 1997 [2], 6.9\% were fully resistant to penicillin. In terms of macrolide resistance, previous studies have reported an overall level of approximately $12 \%$ to $13 \%$ [2-4].

In addition to the high overall rates of resistance reported previously, the prevalence of lower RTIs caused by these pathogens is known to vary greatly depending on geographic location, and the same is true for the rates of resistance to antibacterial drugs. Important differences exist between the rates of antibacterial resistance in Latin America countries and even between the rates in different cities within each country [2-4]. Furthermore, multiple-resistance to the $\beta$-lactams and other antimicrobial agents, such as the cephalosporins and the macrolide-lincosamidestreptogramin (MLS) group of antibiotics, is a common problem.

These issues have driven research towards improving antibiotic usage strategies, and towards the development of new agents that do not induce or cause selection for resistance, as this will be important for safeguarding future antibiotic efficacy. The ketolides are a new family of agents within the MLS class, designed specifically not to induce MLS $_{B}$ resistance. Telithromycin, the first member of this family to be approved for clinical use, has a well-balanced spectrum of activity covering common RTI bacteria, as well as atypical and intracellular pathogens, such as Chlamydia pneumoniae, Mycoplasma pneumoniae, and Legionella pneumophila [5,6]. The activity of telithromycin also extends to many RTI bacterial strains that have become resistant to existing agents, in particular penicillin-, erythromycin- and fluoroquinolone-resistant S. pneumoniae [5]. The development of newer agents, like the ketolides, needs to be complemented by more rational prescribing of antibiotics in order to help limit the spread of resistance.
With this in mind, continued surveillance of resistance amongst the bacterial species most commonly associated with community-acquired RTIs is essential. PROTEKT (Prospective Resistant Organism Tracking and Epidemiology for the Ketolide Telithromycin) is a longitudinal global surveillance programme, established in 1999 to study the antibacterial susceptibility of common community-acquired RTI pathogens in Northern and Latin America, Eastern and Western Europe, and the Asia Pacific region. We report here on the 1999-2000 PROTEKT findings on antibacterial resistance among community-acquired RTIisolates from participating centers in Latin America, and the activity of the ketolide telithromycin against these isolates.

\section{Materials and Methods}

\section{$\underline{\text { Participating Centers }}$}

During the 1999-2000 winter season, isolates of common pathogens were collected from patients with community-acquired RTIs at 13 participating centers across Latin America. The 13 centers included two centers in Argentina, seven centers in Brazil and four centers in Mexico.

Each center was requested to collect 165 isolates as follows: $20 \mathrm{~S}$. aureus, $25 \mathrm{~S}$. pyogenes, $60 \mathrm{~S}$. pneumoniae, $40 \mathrm{H}$. influenzae and $20 \mathrm{M}$. catarrhalis. In practice the number of isolates collected ranged from 24 to 294 per center, the variation being attributable to a variety of local factors.

\section{Isolate Collection and Storage}

Isolates of $S$. pneumoniae, $H$. influenzae, M. catarrhalis, Streptococcus pyogenes and Staphylococcus aureus were collected from patients with one of the following types of community-acquired RTIs: acute/chronic sinusitis, acute/chronic otitis media, acute/chronic tonsillitis/pharyngitis, bacterial exacerbation of chronic bronchitis, acute exacerbation of chronic obstructive airways disease, or communityacquired pneumonia. Isolates collected from hospitalised patients within 48 hours of admission were also 
included. The following were reasons for exclusion from the analyses: isolates collected from patients with nosocomial lower RTIs or cystic fibrosis, duplicate strains, strains originating from existing collections, and isolates from sputum samples with a poor Gram stain. Demographic data were collected routinely as part of the study.

The four main sources for isolates of RTI pathogens were blood, sputum, bronchoalveolar lavage cultures and middle ear fluid cultures. In addition, four other infection sources were considered acceptable, namely nasopharyngeal swab or aspirate cultures, sinus aspirate cultures, and throat cultures (S. pyogenes only). Following collection, isolates were stored in heavy suspension in horse serum, skimmed milk, Protect ${ }^{\mathrm{TM}}$ tubes, or in the microbank system at a temperature at or below $-20^{\circ} \mathrm{C}$, and subsequently shipped to a central, accredited laboratory (GR Micro Ltd, London, UK) for microbiological investigation. Criteria for isolate identification and re-identification have been described in detail $[7,8]$.

\section{Antibacterial Susceptibility Testing}

A range of $\beta$-lactam, MLS and fluoroquinolone antibacterial agents were tested. Penicillin, amoxycillin/ clavulanate (2:1 ratio), cefuroxime, cefixime, cefaclor, cefpodoxime, clindamycin, erythromycin, clarithromycin, azithromycin, telithromycin, quinupristin-dalfopristin (Synercid, 30:70 ratio), teicoplanin, vancomycin, ciprofloxacin, levofloxacin, moxifloxacin, tetracycline, linezolid and co-trimoxazole (1:19 ratio) were tested against Gram-positive pathogens. All Gram-negative pathogens were tested with the following: ampicillin, amoxycillin, amoxycillin/clavulanate (2:1 ratio), cefditoren, cefprozil, cefuroxime, cefixime, cefdinir, cefaclor, cefpodoxime, erythromycin, clarithromycin, azithromycin, telithromycin, ciprofloxacin, levofloxacin, moxifloxacin, tetracycline, chloramphenicol, linezolid and co-trimoxazole (1:19 ratio).

The central laboratory determined minimum inhibitory concentrations (MICs) using lyophilised microtitre plates (Sensititre system, Trek Diagnostics) with an inoculum of $5 \times 10^{4}$ colony forming units (CFU) in $100 \mathrm{ml}$ media. Susceptibility tests were conducted according to National Committee for Clinical Laboratory Standards (NCCLS) guidelines for the broth microdilution method [9]. After overnight incubation with the test antibacterial aerobically at $37^{\circ} \mathrm{C}$, MIC endpoints were read as the lowest concentration of agent that totally inhibited macroscopically visible growth of the inoculum.

Published NCCLS breakpoints [10] were used to define susceptibility. Since NCCLS breakpoints are not yet available for the new ketolide telithromycin, only $\mathrm{MIC}_{50}, \mathrm{MIC}_{90}$ and MIC range were reported.

\section{Determination of Bacterial Resistance Mechanisms}

$\beta$-lactamase production was determined in $M$. catarrhalis and $H$. influenzae, using the chromogenic cephalosporin (nitrocefin) test (Unipath Ltd, Basingstoke, UK). All macrolide-resistant S. pneumoniae isolates were processed and tested for the presence of erm(A), erm(A) subclass erm(TR), $\operatorname{erm}(\mathrm{B}), \operatorname{erm}(\mathrm{C})$, and $m e f(\mathrm{~A})$ genes using a multiplex rapid-cycle polymerase chain reaction (PCR) with microwell-format probe hybridisation, as described previously [11]. A similar methodology was also employed for $S$. pyogenes to test for the presence of $\operatorname{erm}(\mathrm{A}), \operatorname{erm}(\mathrm{A})$ subclass $\operatorname{erm}(\mathrm{TR}), \operatorname{erm}(\mathrm{B}), \operatorname{erm}(\mathrm{C})$, and $m e f(\mathrm{~A})$.

\section{Results}

A total of 1,806 isolates were collected (Table 1). Of these, $453(25.1 \%)$ were collected from paediatric patients (<14 years of age); this frequency ranged from 30/200 (15\%) in Argentina, 123/646 (19.0\%) in Mexico to 300/960 (31.3\%) in Brazil.

$\underline{\text { Susceptibility of } S \text {. pneumoniae Isolates }}$

A total of 518 S. pneumoniae isolates were available for microbiological investigation in Latin America (Argentina, $n=55$; Brazil, $n=260$; Mexico, $\mathrm{n}=203$ ) (Table 1). Overall, $218(42.1 \%)$ of the S. pneumoniae isolates had reduced susceptibility to 
penicillin (MIC >0.06 mg/L), 79 (15.3\%) of which were fully resistant (MIC $\geq 2 \mathrm{mg} / \mathrm{L}$ (Table 2). The highest proportion of fully resistant isolates was observed in Mexico, where resistance reached $76.5 \%$ in Mexico City (24.1\% overall). While the overall proportion of fully resistant isolates was relatively low in most centers in Brazil $(8.1 \%$ overall), $30.0 \%$ of the isolates from Brasilia were fully resistant to penicillin. As shown in Table 3, the pattern of pneumococcal resistance to penicillin was also seen for the other $\beta$-lactam antibacterials tested across Latin America. The notable exception was amoxycillin/clavulanate, which remained active against $98.7 \%$ of the isolates.

While the number of $S$. pneumoniae isolates with intermediate and full resistance to erythromycin was lower than that observed for penicillin $(15.5 \%$ vs. $42.1 \%$ ), the proportion of isolates with full resistance was identical (15.3\%) (Table 2). MIC distributions for azithromycin, clarithromycin, erythromycin and telithromycin are shown in Figure 1; there were no major differences among the participating countries. As with penicillin, the highest rates of erythromycin resistance were observed in Mexico, reaching 23.0\% in Mexico City Center 31 and 31.2\% in Monterrey (27.6\% overall). In all, 77 erythromycin-resistant S. pneumoniae were analysed for their underlying mechanism of resistance. Of these, 43 tested positive for $m e f(\mathrm{~A})$ and 33 tested positive for $\operatorname{erm}(\mathrm{B})$, while another isolate tested positive for mefA and ermB (Table 4). Differences in the prevalence of resistance, as well as in the mechanisms of erythromycin resistance, were evident among the different centers participating in the study (Tables 2, 3 and 4).

A trend towards decreasing activity of other $\beta$ lactams and the macrolides was observed among $S$. pneumoniae isolates with reduced susceptibility to penicillin (Table 3); almost $100 \%$ of penicillin-resistant strains were resistant to the other $\beta$-lactams, with the exception of amoxycillin/ clavulanate $(3.8 \%)$, and $22.8 \%$ were resistant to erythromycin (Table 3 ). Resistance to co-trimoxazole and tetracycline was also high (45.6\% and $21.8 \%$, respectively) particularly in those resistant to penicillin (Table 3 ). Of the other agents tested, linezolid, teicoplanin and vancomycin were the only ones to which S. pneumoniae remained $100 \%$ susceptible, irrespective of penicillin or erythromycin resistance (susceptibility breakpoints are shown in Table 3 and Figure 2). Against S. pneumoniae, telithromycin had an $\mathrm{MIC}_{90}$ of $0.06 \mathrm{mg} / \mathrm{L}$, with $100 \%$ of the isolates being susceptible to this agent at an MIC of $0.5 \mathrm{mg} / \mathrm{L}$.

The overall prevalence of resistance to the fluoroquinolones was low $(0.8 \%)$, with one isolate $(0.4 \%)$ in Brazil and three isolates $(1.5 \%)$ in Mexico being fully resistant to levofloxacin (MIC $\geq 8 \mathrm{mg} / \mathrm{L}$ ) (Table 2). These fluoroquinolone-resistant isolates also had reduced susceptibility to many of the $\beta$-lactam and MLS antibacterial agents tested, butretained full susceptibility to clindamycin, linezolid, teicoplanin, vancomycin and the ketolide telithromycin at an MIC of $0.5 \mathrm{mg} / \mathrm{L}$.

$\underline{\text { Susceptibility of } H \text {. influenzae and } M \text {. catarrhalis }}$ Isolates

Of the $520 \mathrm{H}$. influenzae isolates collected in Latin America (Table 1), 88 (16.9\%) produced $\beta$-lactamase, ranging from $11.0 \%$ in Brazil, to $19.2 \%$ in Argentina, and to $24.6 \%$ in Mexico. These isolates were resistant to ampicillin and amoxycillin, although no $\beta$-lactamasenegative, ampicillin-resistant $H$. influenzae isolates were detected (Table 5).

$\beta$-lactamase-producing $H$. influenzae were also found to be resistant to the following antibacterials: cefaclor $(2.3 \%)$, cefprozil $(4.5 \%)$, chloramphenicol $(11.4 \%)$ and tetracycline (12.5\%), although the overall rate of resistance to these agents among $H$. influenzae isolates remained low ( $<3 \%$, Table 5). In contrast, resistance to co-trimoxazole was high in both $\beta$ lactamase-positive and -negative strains; resistance was reported for $34.8 \%$ of the $H$. influenzae isolates ( $\beta$-lactamase-negative, $30.1 \%$; $\beta$-lactamase-positive, $58.0 \%)$. All other agents retained high activity against $H$. influenzae (Table 5). Importantly, $H$. influenzae remained susceptible to the ketolide telithromycin and the macrolide azithromycin (Figure 3), irrespective of $\beta$-lactamase production. The $\mathrm{MIC}_{90}$ and range for telithromycin against $H$ influenzae were 2 and 0.002 $4 \mathrm{mg} / \mathrm{L}$, respectively. 
Table 1. Number of isolates of respiratory pathogens collected from Latin America during the PROTEKT study, 1999-2000

\section{No. of isolates}

\begin{tabular}{lccccc} 
& S. pneumoniae & H. influenzae & M. catarrhalis & S. aureus & S. pyogenes \\
\hline Argentina (n=200) & 55 & 52 & 40 & 20 & 33 \\
Brazil (n=960) & 260 & 273 & 71 & 211 & 145 \\
Mexico (n=646) & 203 & 195 & 29 & 120 & 99 \\
Total $(\mathbf{n = 1 8 0 6 )}$ & $\mathbf{5 1 8}$ & $\mathbf{5 2 0}$ & $\mathbf{1 4 0}$ & $\mathbf{3 5 1}$ & $\mathbf{2 7 7}$ \\
\hline
\end{tabular}

Figure 1a. PROTEKT 1999-2000: MIC distributions for macrolides and telithromycin against S. pneumoniae in 1999-2000 from Argentina

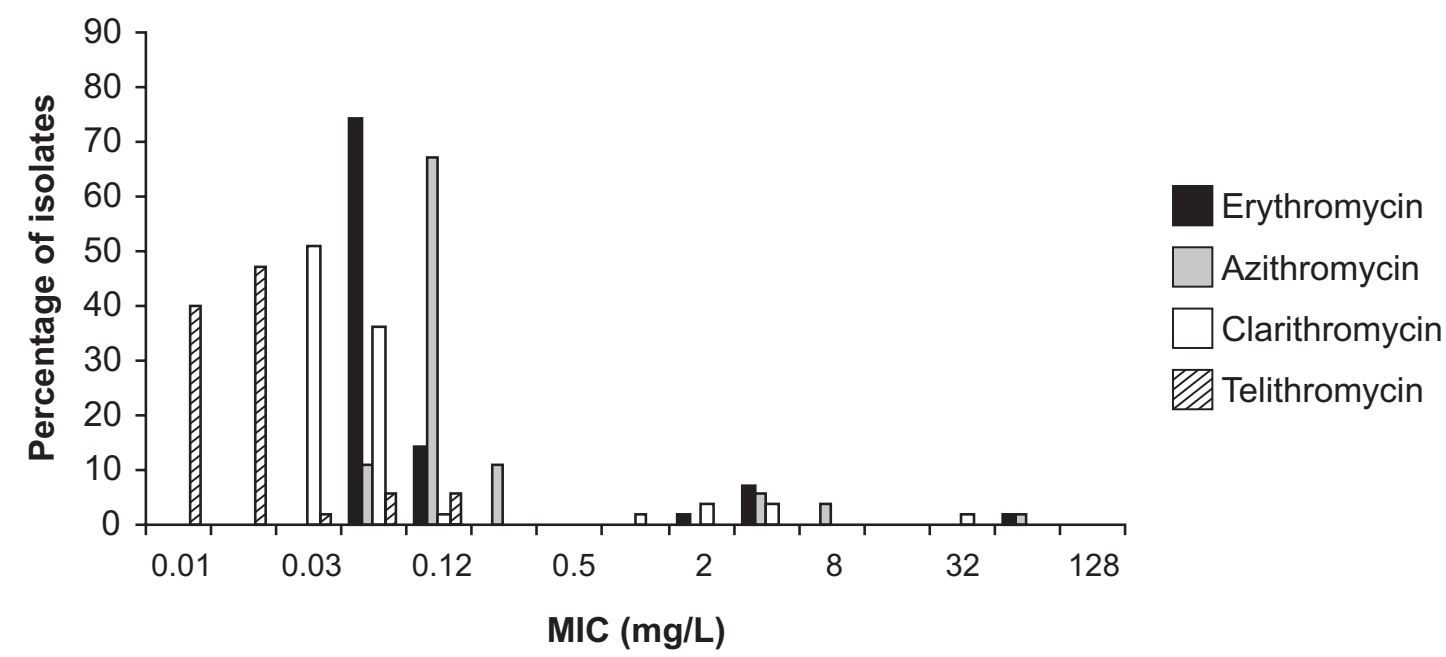

Figure 1b. PROTEKT 1999-2000: MIC distributions for macrolides and telithromycin against S. pneumoniae in 1999-2000 from Brazil

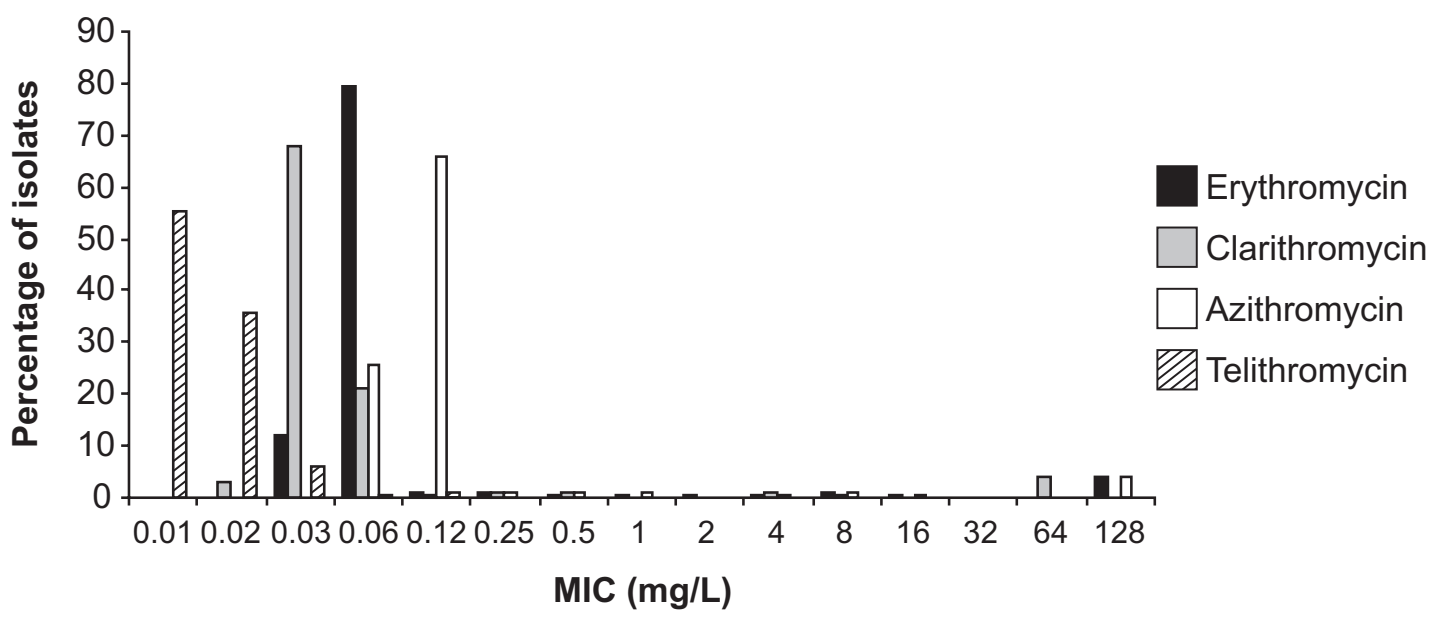


Figure 1c. PROTEKT 1999-2000: MIC distributions for macrolides and telithromycin against S. pneumoniae in 1999-2000 from Mexico

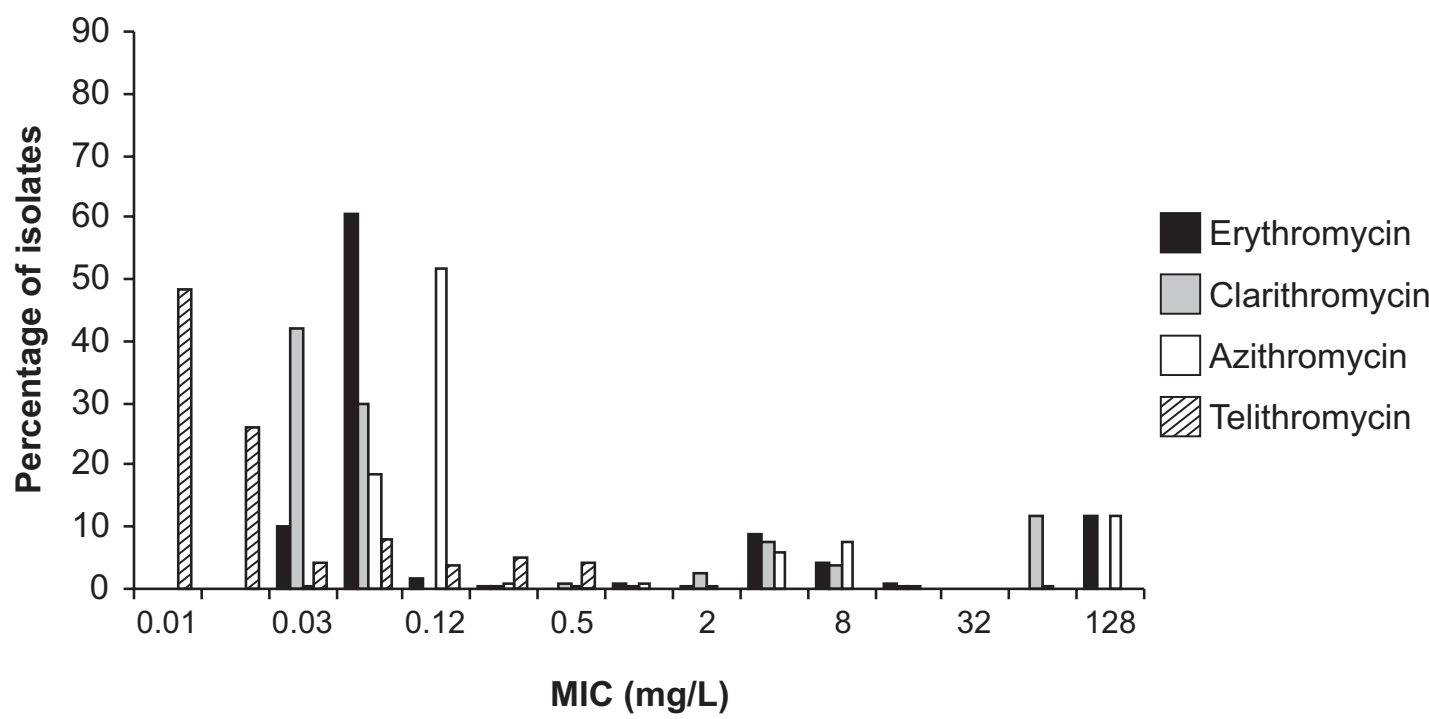

$\beta$-lactamase was produced by almost all of the 140 isolates of $M$. catarrhalis collected in Latin America, including $97.5 \%$ of the isolates from Argentina, $98.6 \%$ of those from Brazil, and $100 \%$ from Mexico $(98.6 \%$ overall). With the exception of ampicillin and amoxycillin, for which the overall rates of resistance were $82.9 \%$ and $87.1 \%$, respectively, almost all of the $M$. catarrhalis isolates collected in Latin America were fully susceptible to the other antibacterials (Table 5). However, a trend towards a decreasing susceptibility to other $\beta$-lactams (cefaclor, cefdinir and cefprozil), and also co-trimoxazole, was observed. MIC values for telithromycin against $M$. catarrhalis ranged from 0.008 to $0.25 \mathrm{mg} / \mathrm{L}$ $\left(\mathrm{MIC}_{50}: 0.06 \mathrm{mg} / \mathrm{L}, \mathrm{MIC}_{90}, 0.12 \mathrm{mg} / \mathrm{L}\right)$.

\section{$\underline{\text { Susceptibility of } S \text {. pyogenes Isolates }}$}

In total, 277 isolates of $S$. pyogenes were submitted in Latin America and underwent microbiological investigation (Table 1). As expected, S. pyogenes remained fully susceptible to penicillin, and hence by NCCLS criteria [10] to other $\beta$-lactams.

Twenty-four (8.7\%) isolates were found to be resistant to erythromycin and azithromycin and $22(7.9 \%)$ were resistant to clarithromycin, of which 18 tested positive for mef(A), 5 for erm(TR) and one isolate tested positive for
ermB. The prevalence of macrolide resistance among S. pyogenes varied between countries (5.5\% in Brazil, $11.1 \%$ in Mexico and $12.1 \%$ in Argentina). In addition, $18.4 \%$ of the isolates were fully resistant to tetracycline (8.1\% in Mexico, 21.2\% in Argentina and $24.8 \%$ in Brazil). Against $S$. pyogenes, telithromycin had an $\mathrm{MIC}_{90}$ of $0.015 \mathrm{mg} / \mathrm{L}$, with $100 \%$ of the isolates being susceptible to this agent at an $\mathrm{MIC}$ of $\leq 0.5 \mathrm{mg} / \mathrm{L}$ ).

\section{Susceptibility of $S$. aureus Isolates}

Overall, 351 isolates of $S$. aureus were collected from centers in Latin America (Table 1). Methicillinresistant $S$. aureus (MRSA) isolates from Argentina (15\%), Mexico (20\%) and Brazil (31.3\%) were detected, giving an overall MRSA rate of $26.5 \%$ in Latin America. However, all isolates were fully susceptible to vancomycin, linezolid and teicoplanin, irrespective of methicillin susceptibility. Eighteen percent of the $S$. aureus isolates were resistant to cotrimoxazole; this figure increased to $35.5 \%$ among MRSA. Methicillin-susceptible strains of $S$. aureus (MSSA) were also highly susceptible to telithromycin (97.7\%), with a maximum $\mathrm{MIC}_{90}$ of $0.06 \mathrm{mg} / \mathrm{L}$, although 91 of the 93 MRSA strains $(97.9 \%$ ) were found to be resistant to this agent. 


\begin{tabular}{|c|c|c|c|c|c|c|c|c|c|c|}
\hline \multirow[b]{2}{*}{ Center } & \multirow[b]{2}{*}{$\begin{array}{c}\text { Total } \\
\text { no. of isolates }\end{array}$} & \multicolumn{3}{|c|}{ Penicillin susceptibility } & \multicolumn{3}{|c|}{ Erythromycin susceptibility } & \multicolumn{3}{|c|}{ Levofloxacin susceptibility } \\
\hline & & $\begin{array}{c}\text { SUSC } \\
\text { no. }(\%)\end{array}$ & $\begin{array}{c}\text { INT } \\
\text { no. }(\%)\end{array}$ & $\begin{array}{c}\text { RES } \\
\text { no. }(\%)\end{array}$ & $\begin{array}{c}\text { SUSC } \\
\text { no. }(\%)\end{array}$ & $\begin{array}{c}\text { INT } \\
\text { no. }(\%)\end{array}$ & $\begin{array}{c}\text { RES } \\
\text { no. }(\%)\end{array}$ & $\begin{array}{c}\text { SUSC } \\
\text { no. }(\%)\end{array}$ & $\begin{array}{c}\text { INT } \\
\text { no. }(\%)\end{array}$ & $\begin{array}{c}\text { RES } \\
\text { no. }(\%)\end{array}$ \\
\hline Argentina & 55 & $40 \quad(72.7)$ & $6(10.9)$ & $9(16.4)$ & $49(89.1)$ & $0(0.0)$ & $6(10.9)$ & $55(100.0)$ & $0(0.0)$ & $0(0.0)$ \\
\hline Buenos Aires 50 & 52 & 38 (73.1) & $5 \quad(9.6)$ & $9(17.3)$ & $48 \quad(92.3)$ & $0(0.0)$ & $4 \quad(7.7)$ & $52(100.0)$ & $0(0.0)$ & $0(0.0)$ \\
\hline Buenos Aires 51 & 3 & $2(66.7)$ & 1 (33.3) & $\begin{array}{ll}0 & (0.0)\end{array}$ & $1(33.3)$ & $0(0.0)$ & $2(66.7)$ & $3(100.0)$ & $0(0.0)$ & $0(0.0)$ \\
\hline Brazil & 260 & $172(66.2)$ & $67 \quad(25.8)$ & (8.1) & $242(93.1)$ & $1(0.4)$ & $17 \quad(6.5)$ & 259 (99.6) & $0(0.0)$ & $1(0.4)$ \\
\hline Florianópolis 41 & 52 & 44 (84.6) & $6(11.5)$ & (3.8) & $46(88.5)$ & $1(1.9)$ & (9.6) & $52(100.0)$ & $0(0.0)$ & $0(0.0)$ \\
\hline Brasilia 45 & 20 & $11 \quad(55.0)$ & $3(15.0)$ & $6(30.0)$ & $17(85.0)$ & $0(0.0)$ & $3(15.0)$ & $20(100.0)$ & $0(0.0)$ & $0(0.0)$ \\
\hline São Paulo 40 & 58 & 34 (58.6) & $21 \quad(36.2)$ & $3 \quad(5.2)$ & $57(98.3)$ & $0(0.0)$ & $1 \quad(1.7)$ & $58(100.0)$ & $0(0.0)$ & $0(0.0)$ \\
\hline São Paulo 42 & 70 & 36 (51.4) & 27 (38.6) & $7(10.0)$ & $64 \quad(91.4)$ & $0(0.0)$ & (8.6) & 69 (98.6) & $0(0.0)$ & $1(1.4)$ \\
\hline São Paulo 44 & 4 & $4(100.0)$ & $\begin{array}{ll}0 & (0.0)\end{array}$ & $\begin{array}{ll}0 & (0.0)\end{array}$ & $4(100.0)$ & $0(0.0)$ & $(0.0)$ & $4(100.0)$ & $0(0.0)$ & $0(0.0)$ \\
\hline São Paulo 46 & 56 & $43 \quad(76.8)$ & $10(17.9)$ & $3 \quad(5.4)$ & 54 (96.4) & $0(0.0)$ & 2 (3.6) & $56(100.0)$ & $0(0.0)$ & $0(0.0)$ \\
\hline Mexico & 203 & $88 \quad(43.4)$ & $66(32.5)$ & $49(24.1)$ & $147 \quad(72.4)$ & $0(0.0)$ & 56 (27.6) & $200(98.5)$ & $0(0.0)$ & $3(1.5)$ \\
\hline México City 31 & 61 & $27 \quad(44.3)$ & $22(36.1)$ & $12(19.7)$ & $47 \quad(77.1)$ & $0(0.0)$ & $14(23.0)$ & $61(100.0)$ & $0(0.0)$ & $0(0.0)$ \\
\hline Mexico City 32 & 17 & $2(11.8)$ & $2(11.8)$ & $13(76.5)$ & 14 (82.4) & $0(0.0)$ & 3 (17.6) & $17(100.0)$ & $0(0.0)$ & $0(0.0)$ \\
\hline Monterrey 33 & 125 & $59(47.2)$ & $42 \quad(33.6)$ & $24(19.2)$ & $86(68.8)$ & $0(0.0)$ & $39(31.2)$ & 122 (97.6) & $0(0.0)$ & $3(2.4)$ \\
\hline Latin America total & 518 & 300 (57.9) & $139(26.8)$ & $79(15.3)$ & $438(84.6)$ & $1(0.2)$ & $79(15.3)$ & $514(99.2)$ & $0(\mathbf{0 . 0})$ & $4(0.8)$ \\
\hline
\end{tabular}

Penicillin breakpoints: susceptible, MIC $\leq 0.06 \mathrm{mg} / \mathrm{L}$; intermediate, MIC 0.12-1 mg/L; resistant, MIC $\geq 2 \mathrm{mg} / \mathrm{L}$.

Erythromycin breakpoints: susceptible, MIC $\leq 0.25 \mathrm{mg} / \mathrm{L}$; intermediate, MIC $0.5 \mathrm{mg} / \mathrm{L}$; resistant, MIC $\geq 1 \mathrm{mg} / \mathrm{L}$.

Levofloxacin breakpoints: susceptible, MIC $\leq 2 \mathrm{mg} / \mathrm{L}$; intermediate, MIC $4 \mathrm{mg} / \mathrm{L} ;$ resistant, MIC $\geq 8 \mathrm{mg} / \mathrm{L}$. 
Figure 2a. PROTEKT 1999-2000: MIC distributions for telithromycin against $S$. pneumoniae isolated from Latin America in 1999-2000, according to penicillin susceptibility

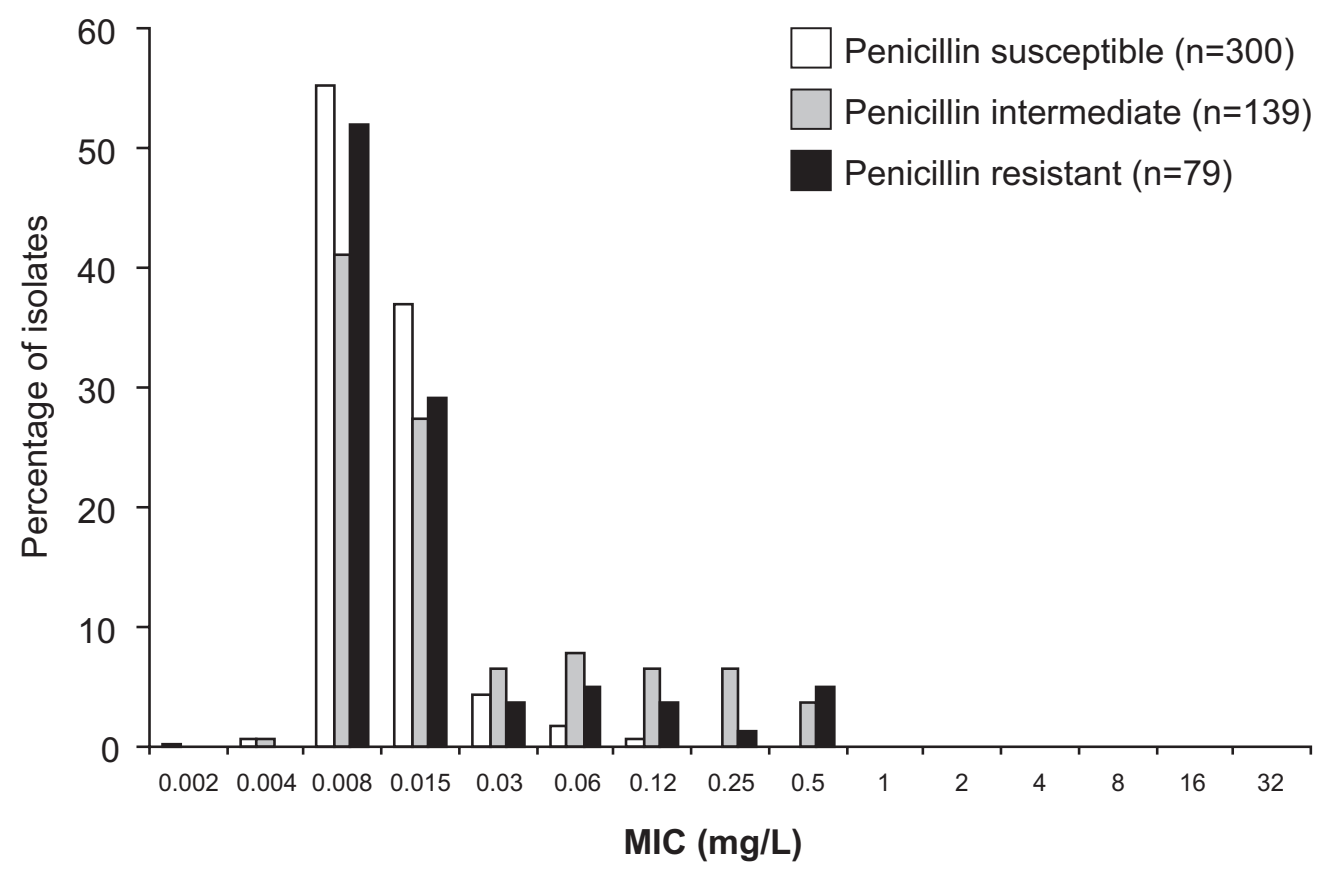

Figure 2b. PROTEKT 1999-2000: MIC distributions for telithromycin against S. pneumoniae isolated from Latin America in 1999-2000, according to erythromycin susceptibility

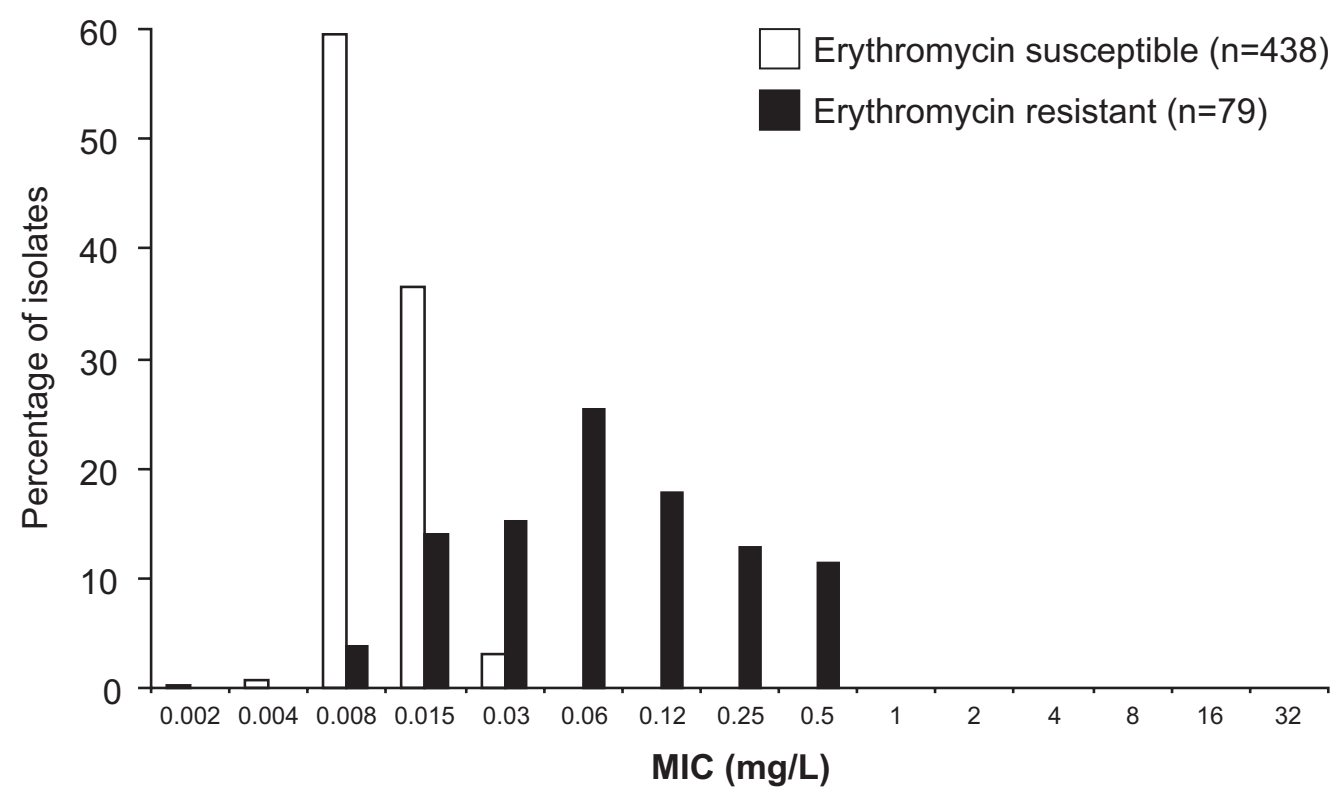


Table 3. PROTEKT 1999-2000:Activity of various antibacterials against clinical isolates of penicillin-susceptible $(\mathrm{n}=300)$, -intermediate $(\mathrm{n}=139)$ and -resistant $(\mathrm{n}=79)$ S. pneumoniae in Latin America, 1999-2000

\begin{tabular}{|c|c|c|c|c|c|}
\hline \multirow[b]{2}{*}{ Antibacterial } & \multicolumn{3}{|c|}{ Susceptibility } & \multicolumn{2}{|c|}{ MIC parameters } \\
\hline & SUSC (\%) & INT (\%) & $\operatorname{RES}(\%)$ & $\mathrm{MIC}_{90}(\mathrm{mg} / \mathrm{L})$ & MIC range (mg/L) \\
\hline \multicolumn{6}{|l|}{ Penicillin } \\
\hline All isolates & $300(57.9)$ & $139(26.8)$ & $79(15.3)$ & 2 & $0.008-8$ \\
\hline PenS & $300(100)$ & $\begin{array}{ll}0 & (0.0)\end{array}$ & $0 \quad(0.0)$ & 0.06 & $0.008-0.06$ \\
\hline PenI & $0(0.0)$ & 139 (100) & $0 \quad(0.0)$ & 1 & $0.12-1$ \\
\hline PenR & $0 \quad(0.0)$ & $0 \quad(0.0)$ & $79(100)$ & 4 & $2-8$ \\
\hline \multicolumn{6}{|c|}{ Amoxycillin/clavulanate } \\
\hline All isolates & $511(98.7)$ & $4(0.8)$ & $3(0.6)$ & 2 & $0.008-8$ \\
\hline PenS & $300(100)$ & $0 \quad(0.0)$ & $0 \quad(0.0)$ & 0.03 & $0.008-0.06$ \\
\hline PenI & $139(100)$ & $0 \quad(0.0)$ & $0 \quad(0.0)$ & 1 & $0.03-2$ \\
\hline PenR & $72(91.1)$ & $4 \quad(5.1)$ & $3(3.8)$ & 2 & $1-8$ \\
\hline \multicolumn{6}{|l|}{ Cefaclor } \\
\hline All isolates & $330(63.7)$ & $55(10.6)$ & $133(25.7)$ & 64 & $0.5-128$ \\
\hline $\operatorname{Pen}^{\mathrm{S}}$ & $280(93.3)$ & $20 \quad(6.7)$ & $0 \quad(0.0)$ & 1 & $0.5-2$ \\
\hline $\operatorname{Pen}^{\mathrm{I}}$ & $50(36.0)$ & $35(25.2)$ & $54(38.9)$ & 32 & $0.5-128$ \\
\hline $\operatorname{Pen}^{\mathrm{R}}$ & $0 \quad(0.0)$ & $0 \quad(0.0)$ & 79 (100) & $>64$ & $32-128$ \\
\hline \multicolumn{6}{|l|}{ Cefixime? } \\
\hline All isolates & $300(57.9)$ & & & 32 & $0.12-128$ \\
\hline Pen $^{S}$ & $300(100)$ & & & 1 & $0.12-8$ \\
\hline Pen $^{I}$ & $0(0.0)$ & & & 16 & $0.25-128$ \\
\hline $\operatorname{Pen}^{\mathrm{R}}$ & $0 \quad(0.0)$ & & & 64 & $16-128$ \\
\hline \multicolumn{6}{|l|}{ Cefpodoxime } \\
\hline All isolates & $406(78.4)$ & $19 \quad(3.7)$ & $93(18.0)$ & 2 & $0.12-32$ \\
\hline $\operatorname{Pen}^{S}$ & $300(100)$ & $0 \quad(0.0)$ & $0 \quad(0.0)$ & 0.12 & $0.12-0.5$ \\
\hline $\operatorname{Pen}^{\mathrm{I}}$ & $106(76.3)$ & $16(11.5)$ & $17(12.2)$ & 2 & $0.12-32$ \\
\hline $\operatorname{Pen}^{\mathrm{R}}$ & $0(0.0)$ & 3 (3.8) & $76(96.2)$ & 4 & 1-16 \\
\hline \multicolumn{6}{|l|}{ Cefuroxime } \\
\hline All isolates & $399(77.0)$ & $14 \quad(2.7)$ & $105(20.3)$ & 4 & $0.015-16$ \\
\hline $\operatorname{Pen}^{\mathrm{S}}$ & $300(100)$ & $0 \quad(0.0)$ & $\begin{array}{ll}0 & (0.0)\end{array}$ & 0.12 & $0.015-1$ \\
\hline $\operatorname{Pen}^{\mathrm{I}}$ & 99 (71.2) & $14(10.1)$ & $26(18.7)$ & 4 & $0.03-16$ \\
\hline $\operatorname{Pen}^{\mathrm{R}}$ & $0 \quad(0.0)$ & $0 \quad(0.0)$ & 79 (100) & 8 & 4-16 \\
\hline \multicolumn{6}{|l|}{ Azithromycin } \\
\hline All isolates & $439(84.8)$ & $4 \quad(0.8)$ & $75(14.5)$ & 8 & $0.03-128$ \\
\hline $\operatorname{Pen}^{S}$ & $285(95.0)$ & $1 \quad(0.3)$ & $14(4.7)$ & 0.12 & $0.06-128$ \\
\hline $\operatorname{Pen}^{\mathrm{I}}$ & $93(66.9)$ & $1 \quad(0.7)$ & $45(32.4)$ & $>64$ & $0.06-128$ \\
\hline $\operatorname{Pen}^{\mathrm{R}}$ & $61(77.2)$ & $2 \quad(2.5)$ & $16(20.3)$ & 8 & $0.03-128$ \\
\hline \multicolumn{6}{|l|}{ Clarithromycin } \\
\hline All isolates & 439 (84.8) & $4 \quad(0.8)$ & $75(14.5)$ & 4 & $0.015-64$ \\
\hline $\operatorname{Pen}^{\mathrm{S}}$ & $285(95.0)$ & $1 \quad(0.3)$ & 14 (4.7) & 0.06 & $0.015-64$ \\
\hline $\operatorname{Pen}^{I}$ & $93(66.9)$ & $1 \quad(0.7)$ & $45(32.4)$ & $>32$ & $0.015-64$ \\
\hline $\operatorname{Pen}^{\mathrm{R}}$ & $61(77.2)$ & $2 \quad(2.5)$ & $16(20.3)$ & 8 & $0.03-64$ \\
\hline \multicolumn{6}{|l|}{ Erythromycin } \\
\hline All isolates & 438 (84.6) & $1 \quad(0.2)$ & $79(15.3)$ & 4 & $0.03-128$ \\
\hline $\operatorname{Pen}^{S}$ & $285(95.0)$ & $\begin{array}{ll}0 & (0.0)\end{array}$ & $15(5.0)$ & 0.06 & $0.03-128$ \\
\hline $\operatorname{Pen}^{I}$ & $92(66.2)$ & $1 \quad(0.7)$ & $46(33.1)$ & $>64$ & $0.03-128$ \\
\hline $\operatorname{Pen}^{\mathrm{R}}$ & $61(77.2)$ & $0 \quad(0.0)$ & $18(22.8)$ & 8 & $0.03-128$ \\
\hline
\end{tabular}




\begin{tabular}{|c|c|c|c|c|c|}
\hline \multirow[b]{2}{*}{ Antibacterial } & \multicolumn{3}{|c|}{ Susceptibility } & \multicolumn{2}{|c|}{ MIC parameters } \\
\hline & SUSC (\%) & INT (\%) & $\operatorname{RES}(\%)$ & $\mathrm{MIC}_{90}(\mathrm{mg} / \mathrm{L})$ & $\mathrm{MIC}$ range $(\mathrm{mg} / \mathrm{L})$ \\
\hline \multicolumn{6}{|l|}{ Telithromycin } \\
\hline All isolates & $\mathrm{N} / \mathrm{A}^{\#}$ & N/A & N/A & 0.06 & $0.002-0.5$ \\
\hline Pen ${ }^{\mathrm{s}}$ & & & & 0.015 & $0.002-0.12$ \\
\hline Pen ${ }^{I}$ & & & & 0.25 & $0.004-0.5$ \\
\hline Pen ${ }^{\mathrm{R}}$ & & & & 0.12 & $0.008-0.5$ \\
\hline \multicolumn{6}{|l|}{ Levofloxacin } \\
\hline All isolates & 514 (99.2) & $\begin{array}{ll}0 & (0.0)\end{array}$ & $4(0.8)$ & 1 & $0.5-16$ \\
\hline Pen ${ }^{\mathrm{S}}$ & $298(99.3)$ & $\begin{array}{ll}0 & (0.0)\end{array}$ & $2(0.7)$ & 1 & $0.5-16$ \\
\hline Pen ${ }^{\mathrm{I}}$ & $139(100)$ & $\begin{array}{ll}0 & (0.0)\end{array}$ & $0(0.0)$ & 1 & $0.5-2$ \\
\hline Pen $^{\mathrm{R}}$ & 77 (97.5) & $\begin{array}{ll}0 & (0.0)\end{array}$ & $2(2.5)$ & 1 & $0.5-16$ \\
\hline \multicolumn{6}{|l|}{ Moxifloxacin } \\
\hline All isolates & $514(99.2)$ & $2(0.4)$ & $2(0.4)$ & 0.25 & $0.06-4$ \\
\hline Pen ${ }^{\mathrm{s}}$ & $298(99.3)$ & $1 \quad(0.3)$ & $1(0.3)$ & 0.25 & $0.06-4$ \\
\hline Pen ${ }^{\mathrm{I}}$ & $139(100)$ & $\begin{array}{ll}0 & (0.0)\end{array}$ & $0(0.0)$ & 0.25 & $0.06-0.25$ \\
\hline Pen $^{\mathrm{R}}$ & 77 (97.5) & $1(1.3)$ & $1(1.3)$ & 0.25 & $0.06-4$ \\
\hline \multicolumn{6}{|l|}{ Clindamycin } \\
\hline All isolates & $482(93.1)$ & $\begin{array}{ll}0 & (0.0)\end{array}$ & $36(7.0)$ & 0.12 & $0.03-8$ \\
\hline Pen ${ }^{\mathrm{s}}$ & $289(96.3)$ & $\begin{array}{ll}0 & (0.0)\end{array}$ & $11(3.7)$ & 0.12 & $0.03-8$ \\
\hline Pen ${ }^{\mathrm{I}}$ & $120(86.3)$ & $\begin{array}{ll}0 & (0.0)\end{array}$ & $19(13.7)$ & $>4$ & $0.03-8$ \\
\hline Pen $^{\mathrm{R}}$ & 73 (92.4) & $\begin{array}{ll}0 & (0.0)\end{array}$ & $6(7.6)$ & 0.12 & $0.03-8$ \\
\hline \multicolumn{6}{|l|}{ Co-trimoxazole } \\
\hline All isolates & $189(36.5)$ & $93(18.0)$ & 236 (45.6) & 16 & $0.12-32$ \\
\hline Pen ${ }^{\mathrm{s}}$ & $148(49.3)$ & $72(24.0)$ & $80(26.7)$ & 8 & $0.12-32$ \\
\hline Pen ${ }^{\mathrm{I}}$ & $35(25.2)$ & $18(13.0)$ & $86(61.9)$ & $>16$ & $0.12-32$ \\
\hline Pen $^{\mathrm{R}}$ & $6(7.6)$ & $3(3.8)$ & $70(88.6)$ & $>16$ & $0.25-32$ \\
\hline \multicolumn{6}{|l|}{ Tetracycline } \\
\hline All isolates & $389(75.1)$ & $16 \quad(3.1)$ & $113(21.8)$ & $>16$ & $0.12-32$ \\
\hline Pen ${ }^{\mathrm{s}}$ & $243(81.0)$ & $10 \quad(3.3)$ & 47 (15.7) & 16 & $0.12-32$ \\
\hline Pen ${ }^{\mathrm{I}}$ & $99(71.2)$ & 3 (2.2) & 37 (26.6) & $>16$ & $0.12-32$ \\
\hline Pen $^{\mathrm{R}}$ & $47(59.5)$ & $3 \quad(3.8)$ & $29(36.7)$ & $>16$ & $0.12-32$ \\
\hline \multicolumn{6}{|l|}{ Linezolid } \\
\hline All isolates & $518(100)$ & $\begin{array}{ll}0 & (0.0)\end{array}$ & $0(0.0)$ & 2 & $0.12-2$ \\
\hline Pen ${ }^{\mathrm{S}}$ & $300(100)$ & $\begin{array}{ll}0 & (0.0)\end{array}$ & $0(0.0)$ & 2 & $0.5-2$ \\
\hline Pen ${ }^{\mathrm{I}}$ & $139(100)$ & $\begin{array}{ll}0 & (0.0)\end{array}$ & $0(0.0)$ & 2 & $0.5-2$ \\
\hline Pen $^{\mathrm{R}}$ & $79(100)$ & $\begin{array}{ll}0 & (0.0)\end{array}$ & $0(0.0)$ & 2 & $0.12-2$ \\
\hline \multicolumn{6}{|l|}{ Teicoplanin } \\
\hline All isolates & $518(100)$ & $\begin{array}{ll}0 & (0.0)\end{array}$ & $0(0.0)$ & 0.12 & $0.03-0.25$ \\
\hline Pen ${ }^{\mathrm{s}}$ & $300(100)$ & $\begin{array}{ll}0 & (0.0)\end{array}$ & $0(0.0)$ & 0.12 & $0.03-0.25$ \\
\hline Pen ${ }^{\mathrm{I}}$ & $139(100)$ & $\begin{array}{ll}0 & (0.0)\end{array}$ & $0(0.0)$ & 0.12 & $0.03-0.25$ \\
\hline Pen ${ }^{\mathrm{R}}$ & $79(100)$ & $\begin{array}{ll}0 & (0.0)\end{array}$ & $0(0.0)$ & 0.12 & $0.03-0.12$ \\
\hline \multicolumn{6}{|l|}{ Vancomycin } \\
\hline All isolates & $518(100)$ & $\begin{array}{ll}0 & (0.0)\end{array}$ & $0(0.0)$ & 0.5 & $0.12-1$ \\
\hline Pen ${ }^{\mathrm{s}}$ & $300(100)$ & $\begin{array}{ll}0 & (0.0)\end{array}$ & $0(0.0)$ & 0.5 & $0.25-0.5$ \\
\hline Pen ${ }^{I}$ & $139(100)$ & $0 \quad(0.0)$ & $0(0.0)$ & 0.5 & $0.12-1$ \\
\hline Pen $^{\mathrm{R}}$ & 79 (100) & $0 \quad(0.0)$ & $0(0.0)$ & 0.5 & $0.25-0.5$ \\
\hline
\end{tabular}

NCCLS susceptibility breakpoints [9].

$\mathrm{Pen}^{\mathrm{S}}=$ penicillin G susceptible (MIC $\left.\leq 0.06 \mathrm{mg} / \mathrm{L}\right) ; \mathrm{Pen}^{\mathrm{I}}=$ penicillin $\mathrm{G}$ intermediate (MIC 0.12-1 mg/L); Pen ${ }^{\mathrm{R}}=$ penicillin G resistant $(\mathrm{MIC} \geq 2 \mathrm{mg} / \mathrm{L}$ ). \# Breakpoint for telithromycin not available.

'Cefixime: Susceptibility based on penicillin susceptibility. There are no breakpoints for intermediate or resistant. 
Figure 3a. PROTEKT 1999-2000: MIC distributions for azithromycin, clarithromycin, erythromycin and telithromycin against $H$. influenzae in 1999-2000 from Argentina

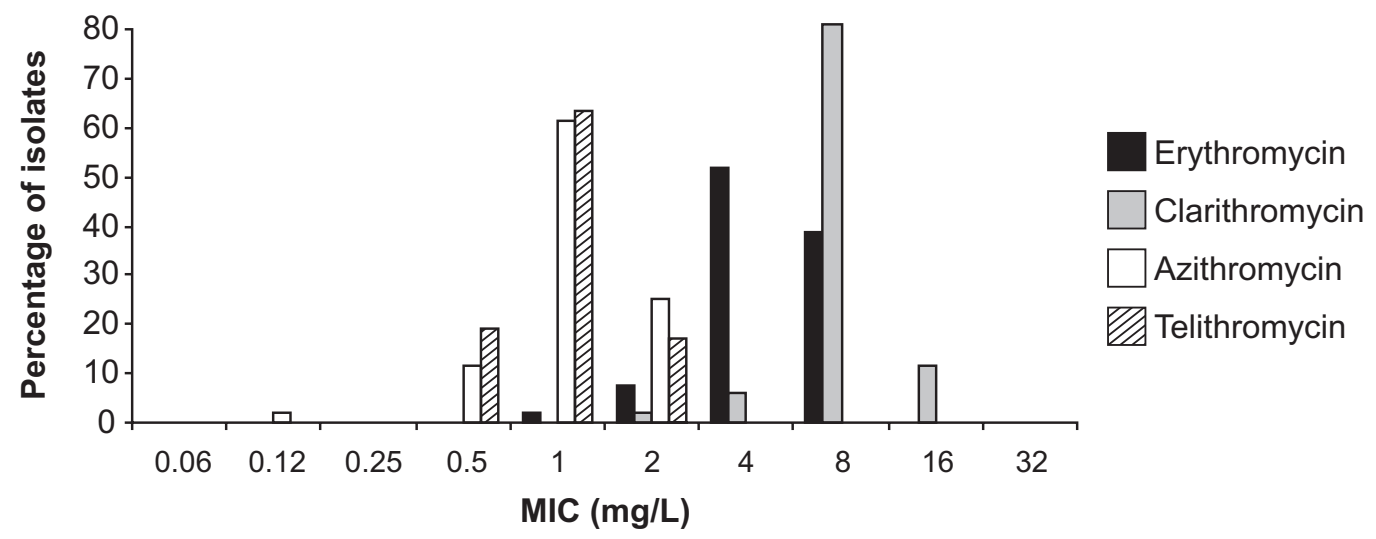

Figure 3b. PROTEKT 1999-2000: MIC distributions for azithromycin, clarithromycin, erythromycin and telithromycin against $H$. influenzae in 1999-2000 from Brazil

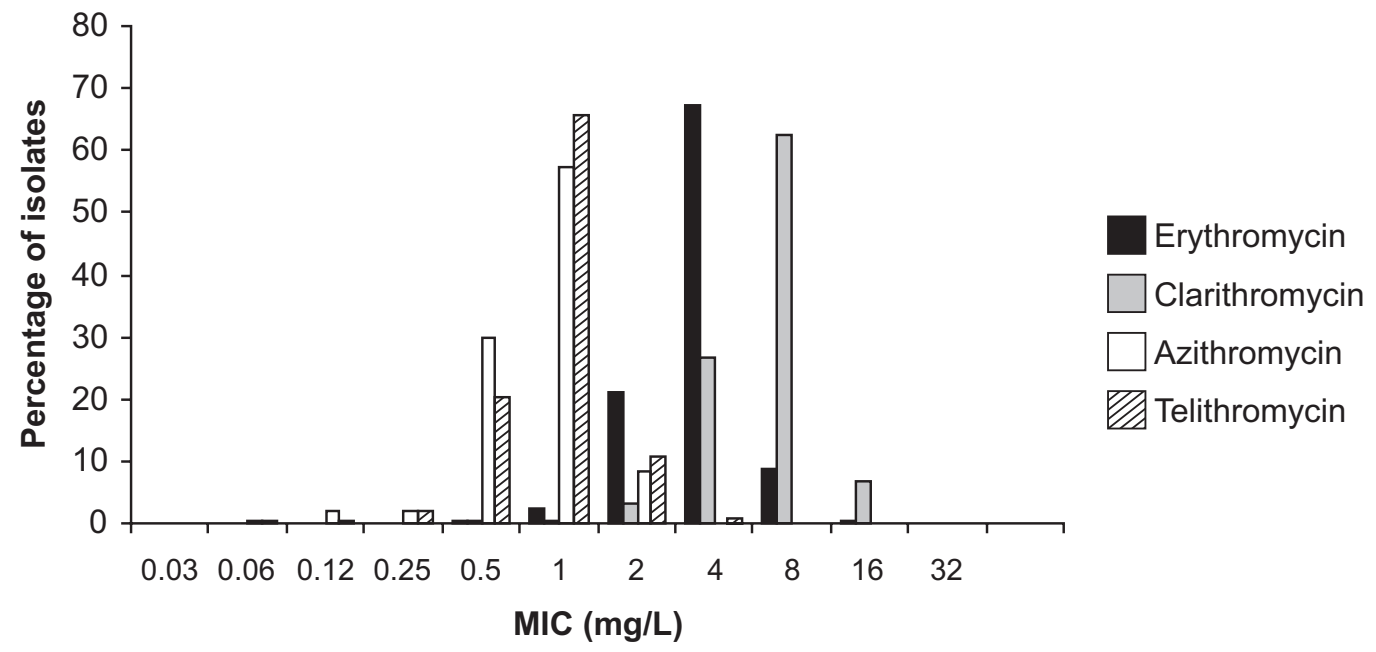

Figure 3c. PROTEKT 1999-2000: MIC distributions for azithromycin, clarithromycin, erythromycin and telithromycin against $H$. influenzae in 1999-2000 from Mexico

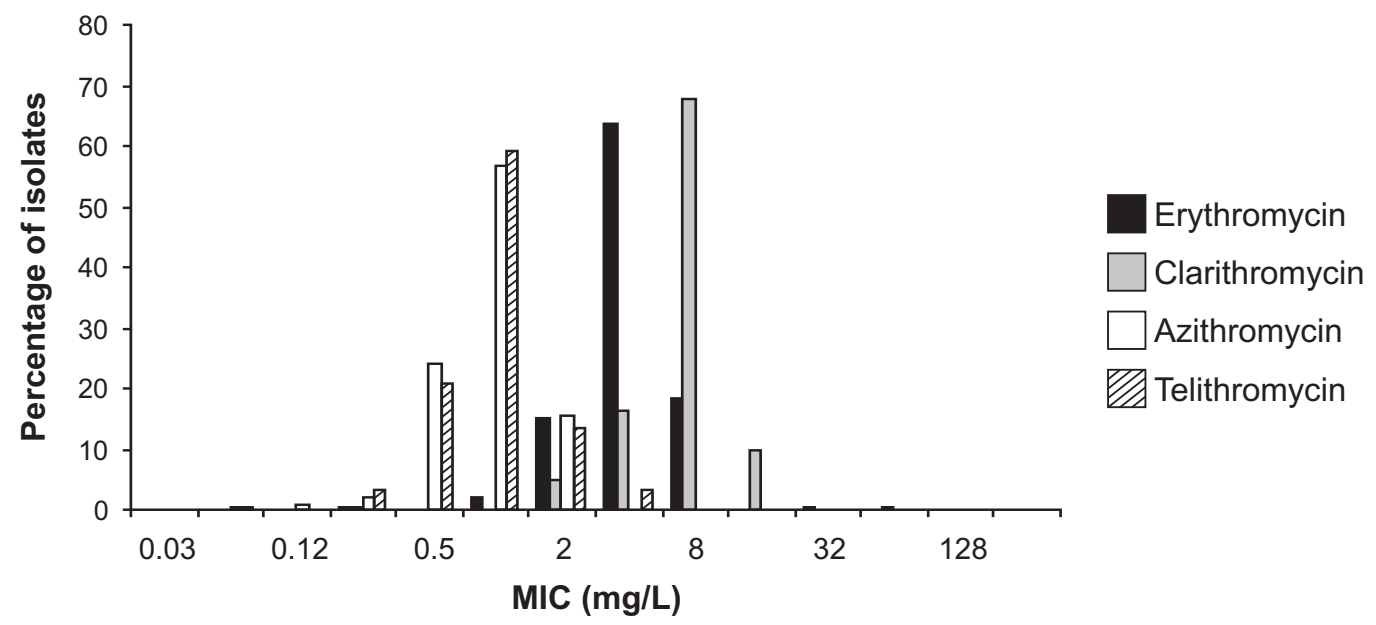


Table 4. PROTEKT 1999-2000: Distribution of macrolide resistance mechanisms among erythromycin-resistant (MIC $\geq 1 \mathrm{mg} / \mathrm{L})$ S. pneumoniae isolated from Latin America in 1999-2000

\begin{tabular}{|c|c|c|c|c|c|}
\hline Center & $\begin{array}{c}\text { Total no.of } \\
\text { isolates tested }\end{array}$ & $\operatorname{Erm}(B)$ & $\operatorname{Mef}(A)$ & $\begin{array}{l}\text { Negative for } \\
\text { mechanisms }\end{array}$ & Total \\
\hline Argentina & 55 & & & & \\
\hline Buenos Aires 50 & 52 & 1 & 3 & & 4 \\
\hline Buenos Aires 51 & 3 & & 2 & & 2 \\
\hline Brazil & 260 & & & & \\
\hline Florianópolis 41 & 52 & 4 & & & 4 \\
\hline Brasilia 45 & 20 & 1 & 2 & & 3 \\
\hline São Paulo 40 & 58 & 1 & & & 1 \\
\hline São Paulo 42 & 70 & 2 & 4 & & 6 \\
\hline São Paulo 43 & 0 & & & & 0 \\
\hline São Paulo 44 & 4 & & & & 0 \\
\hline São Paulo 46 & 56 & 2 & & & 2 \\
\hline Mexico & 203 & & & & \\
\hline Mexico City 31 & 61 & 6 & 8 & & 14 \\
\hline Mexico City 32 & 17 & 3 & & & 3 \\
\hline Monterrey 33 & 125 & 16 & 21 & 1 & 38 \\
\hline Latin America total & 518 & 36 & 40 & 1 & 77 \\
\hline
\end{tabular}

\section{Discussion}

PROTEKT data collected during the period 19992000 indicate that the prevalence of penicillin resistance (MIC $\geq 2 \mathrm{mg} / \mathrm{L}$ ) amongst pneumococci in Latin America is high, with $15.3 \%$ of the isolates being fully resistant and $26.8 \%$ having intermediate resistance to this antibacterial agent. This level of nonsusceptibility appears to be higher than that reported by Odland et al. [1], who analysed over 2,000 pneumococcal isolates as part of the SENTRY programme, of which 264 were from Latin American countries. Among these, 9.9\% were fully resistant to penicillin and $45.8 \%$ were intermediate. Of the 1,100 S. pneumoniae isolates from seven Latin American and Caribbean countries surveyed by the LASER study group in 1997 [2], $23.5 \%$ were nonsusceptible to penicillin (6.9\% resistant/ $16.6 \%$ intermediate). As in the PROTEKT study, a high multiple-resistance pattern towards co-trimoxazole $(44.6 \%)$ was also observed. Similar resistance rates were observed in the Global SMART (GSMART) surveillance study, during which 244 pneumococcal isolates from five Latin American countries were analysed in 1999-2000 [12]. Of these, $28 \%$ were nonsusceptible to penicillin and 5\% were fully resistant. Rates of nonsusceptibility to penicillin varied between the Latin American countries participating in the PROTEKT study (Mexico, 56.6\%; Brazil, 33.9\%; Argentina, 27.3\%). Corresponding rates reported in the LASER study were: Mexico (40.8\%), Brazil (13\%) and Argentina (19.1\%) [2]. Intermediate and full resistance patterns in the PROTEKT study were as follows: Argentina (10.9\% intermediate, $16.4 \%$ resistant), Brazil (25.8\% intermediate; $8.1 \%$ resistant) and Mexico (32.5\% intermediate; $24.1 \%$ resistant). In comparison, in the worldwide Alexander Project conducted between 1996 and 1997, a high rate of intermediate resistance (17.9\%) and a low fully resistant rate of $2.1 \%$ were observed among 95 isolates of $S$. pneumoniae from Brazil; corresponding figures for Mexico were $31.4 \%$ and $15.7 \%$, respectively [3]. In the SIREVA-vigía programme conducted in Latin 
Table 5. PROTEKT 1999-2000:Activity of selected antibacterials against clinical isolates of $H$. influenzae and $M$. catarrhalis from Latin America (1999-2000) according to $\beta$-lactamase status

\begin{tabular}{|c|c|c|c|c|c|c|c|c|}
\hline \multirow[b]{2}{*}{ Antibacterial } & \multicolumn{4}{|c|}{ H. influenzae $(\mathrm{n}=520)$} & \multicolumn{4}{|c|}{ M. catarrhalis $(n=140)$} \\
\hline & $\begin{array}{l}\text { INT } \\
(\%)\end{array}$ & $\begin{array}{l}\text { RES } \\
(\%)\end{array}$ & $\begin{array}{c}\text { MIC90 } \\
(\mathrm{mg} / \mathrm{L})\end{array}$ & $\begin{array}{c}\text { MIC range } \\
(\mathrm{mg} / \mathrm{L})\end{array}$ & $\begin{array}{l}\text { INT* } \\
(\%)\end{array}$ & $\begin{array}{c}\text { RES* } \\
(\%)\end{array}$ & $\begin{array}{c}\text { MIC90 } \\
(\mathrm{mg} / \mathrm{L})\end{array}$ & $\begin{array}{c}\text { MIC range } \\
(\mathrm{mg} / \mathrm{L})\end{array}$ \\
\hline \multicolumn{9}{|l|}{ Ampicillin } \\
\hline All isolates & $0(0.0)$ & $88(16.9)$ & $>16$ & $0.12-32$ & $11(7.9)$ & $116(82.9)$ & 16 & $0.12-32$ \\
\hline$\beta$-lactamase - & $0(0.0)$ & $0(0.0)$ & 0.5 & $0.12-1$ & $0(0.0)$ & $0(0.0)$ & $-{ }^{\mathrm{a}}$ & 0.12 \\
\hline$\beta$-lactamase + & $0(0.0)$ & $88(100)$ & $>16$ & $4-32$ & $116(84.1)$ & $116(84.1)$ & 16 & $0.12-32$ \\
\hline \multicolumn{9}{|l|}{ Amoxycillin } \\
\hline All isolates & $7(1.3)$ & $90(17.3)$ & $>16$ & $0.12-32$ & $7(5.0)$ & $122(87.1)$ & $>16$ & $0.12-32$ \\
\hline$\beta$-lactamase - & $6(1.4)$ & $3(0.7)$ & 0.5 & $0.12-4$ & $0(0.0)$ & $0(0.0)$ & $-{ }^{\mathrm{a}}$ & 0.12 \\
\hline$\beta$-lactamase + & $1(1.1)$ & $87(98.9)$ & $>16$ & $2-32$ & $7(5.1)$ & $122(88.4)$ & $>16$ & $0.12-32$ \\
\hline \multicolumn{9}{|c|}{ Amoxycillin/clavulanate } \\
\hline All isolates & $0(0.0)$ & $0(0.0)$ & 1 & $0.12-4$ & $0(0.0)$ & $0(0.0)$ & 0.25 & $0.12-0.5$ \\
\hline$\beta$-lactamase - & $0(0.0)$ & $0(0.0)$ & 0.5 & $0.12-4$ & $0(0.0)$ & $0(0.0)$ & $\mathrm{a}^{\mathrm{a}}$ & 0.12 \\
\hline$\beta$-lactamase + & $0(0.0)$ & $0(0.0)$ & 1 & $0.25-4$ & $0(0.0)$ & $0(0.0)$ & 0.25 & $0.12-0.5$ \\
\hline \multicolumn{9}{|l|}{ Cefaclor } \\
\hline All isolates & $22(4.2)$ & $2(0.4)$ & 8 & $0.5-64$ & $5(3.6)$ & $0(0.0)$ & 8 & $0.5-16$ \\
\hline$\beta$-lactamase - & $6(1.4)$ & $0(0.0)$ & 4 & $0.5-16$ & $0(0.0)$ & $0(0.0)$ & $-{ }^{\mathrm{a}}$ & $0.5-1$ \\
\hline$\beta$-lactamase + & $16(18.2)$ & $2(2.3)$ & 16 & $1-64$ & $5(3.6)$ & $0(0.0)$ & 8 & $0.5-16$ \\
\hline \multicolumn{9}{|l|}{ Cefdinir } \\
\hline All isolates & $0(0.0)$ & $0(0.0)$ & 0.5 & $0.06-1$ & $0(0.0)$ & $0(0.0)$ & 0.25 & $0.06-1$ \\
\hline$\beta$-lactamase - & $0(0.0)$ & $0(0.0)$ & 0.5 & $0.06-1$ & $0(0.0)$ & $0(0.0)$ & $-^{\mathrm{a}}$ & 0.06 \\
\hline$\beta$-lactamase + & $0(0.0)$ & $0(0.0)$ & 0.5 & $0.06-1$ & $5(3.6)$ & $0(0.0)$ & 0.25 & $0.06-1$ \\
\hline \multicolumn{9}{|l|}{ Cefixime } \\
\hline All isolates & $0(0.0)$ & $0(0.0)$ & 0.06 & $0.008-0.5$ & $0(0.0)$ & $0(0.0)$ & 0.5 & $0.03-0.5$ \\
\hline$\beta$-lactamase - & $0(0.0)$ & $0(0.0)$ & 0.06 & $0.008-0.5$ & $0(0.0)$ & $0(0.0)$ & $-\mathrm{a}^{\mathrm{a}}$ & $0.03-0.06$ \\
\hline$\beta$-lactamase + & $0(0.0)$ & $0(0.0)$ & 0.06 & $0.015-0.5$ & $0(0.0)$ & $0(0.0)$ & 0.5 & $0.03--0.5$ \\
\hline \multicolumn{9}{|l|}{ Cefpodoxime } \\
\hline All isolates & $0(0.0)$ & $0(0.0)$ & 0.12 & $0.015-0.5$ & $0(0.0)$ & $0(0.0)$ & 1 & $0.06-2$ \\
\hline$\beta$-lactamase - & $0(0.0)$ & $0(0.0)$ & 0.12 & $0.015-0.5$ & $0(0.0)$ & $0(0.0)$ & $-^{\mathrm{a}}$ & $0.06-0.12$ \\
\hline$\beta$-lactamase + & $0(0.0)$ & $0(0.0)$ & 0.12 & $0.015-0.5$ & $0(0.0)$ & $0(0.0)$ & 1 & $0.12-2$ \\
\hline \multicolumn{9}{|l|}{ Cefprozil } \\
\hline All isolates & $12(2.3)$ & $4(0.8)$ & 4 & $0.5-128$ & $6(4.3)$ & $1(0.7)$ & 8 & $0.5-32$ \\
\hline$\beta$-lactamase - & $3(0.7)$ & $0(0.0)$ & 4 & $0.5-16$ & $0(0.0)$ & $0(0.0)$ & $-{ }^{\mathrm{a}}$ & 0.5 \\
\hline$\beta$-lactamase + & $9(10.2)$ & $4(4.5)$ & 16 & $0.5-128$ & $6(4.3)$ & $1(0.7)$ & 8 & $0.5-32$ \\
\hline \multicolumn{9}{|l|}{ Cefuroxime } \\
\hline All isolates & $0(0.0)$ & $0(0.0)$ & 1 & $0.12-4$ & $1(0.7)$ & $0(0.0)$ & 2 & $0.12-8$ \\
\hline$\beta$-lactamase - & $0(0.0)$ & $0(0.0)$ & 1 & $0.12-4$ & $0(0.0)$ & $0(0.0)$ & $-^{\mathrm{a}}$ & $0.12-0.5$ \\
\hline$\beta$-lactamase + & $0(0.0)$ & $0(0.0)$ & 2 & $0.25-4$ & $1(0.7)$ & $0(0.0)$ & 2 & $0.5-8$ \\
\hline
\end{tabular}




\begin{tabular}{|c|c|c|c|c|c|c|c|c|}
\hline \multirow[b]{2}{*}{ Antibacterial } & \multicolumn{4}{|c|}{ H. influenzae $(\mathrm{n}=520)$} & \multicolumn{4}{|c|}{ M. catarrhalis $(\mathrm{n}=140)$} \\
\hline & $\begin{array}{l}\text { INT } \\
(\%)\end{array}$ & $\begin{array}{l}\text { RES } \\
(\%)\end{array}$ & $\begin{array}{l}\text { MIC90 } \\
(\mathrm{mg} / \mathrm{L})\end{array}$ & $\begin{array}{c}\text { MIC range } \\
(\mathrm{mg} / \mathrm{L})\end{array}$ & $\begin{array}{l}\text { INT* } \\
(\%)\end{array}$ & $\begin{array}{c}\text { RES* } \\
(\%)\end{array}$ & $\begin{array}{c}\text { MIC90 } \\
(\mathrm{mg} / \mathrm{L})\end{array}$ & $\begin{array}{c}\text { MIC range } \\
(\mathrm{mg} / \mathrm{L})\end{array}$ \\
\hline \multicolumn{9}{|l|}{ Azithromycin } \\
\hline All isolates & $0(0.0)$ & $0(0.0)$ & 2 & $0.06-2$ & $0(0.0)$ & $0(0.0)$ & 0.06 & 0.06 \\
\hline b-lactamase - & $0(0.0)$ & $0(0.0)$ & 2 & $0.06-2$ & $0(0.0)$ & $0(0.0)$ & $-a^{a}$ & 0.06 \\
\hline b-lactamase + & $0(0.0)$ & $0(0.0)$ & 2 & $0.06-2$ & $0(0.0)$ & $0(0.0)$ & 0.06 & 0.06 \\
\hline All isolates & $43(8.3)$ & $1(0.2)$ & 8 & $0.25-64$ & $0(0.0)$ & $0(0.0)$ & 0.25 & 0.25 \\
\hline b-lactamase - & $28(6.5)$ & $1(0.2)$ & 8 & $1-64$ & $0(0.0)$ & $0(0.0)$ & $-a^{a}$ & 0.25 \\
\hline b-lactamase + & $15(17.0)$ & $0(0.0)$ & 16 & $0.24-16$ & $0(0.0)$ & $0(0.0)$ & 0.25 & 0.25 \\
\hline \multicolumn{9}{|l|}{ Telithromycin } \\
\hline All isolates & N/A $\#$ & $\mathrm{~N} / \mathrm{A}^{\#}$ & 2 & $0.002-4$ & $\mathrm{~N} / \mathrm{A}^{\#}$ & $\mathrm{~N} / \mathrm{A}^{\#}$ & 0.12 & $0.008-0.25$ \\
\hline b-lactamase - & & & 2 & $0.002-4$ & & & $-\mathrm{a}^{\mathrm{a}}$ & $0.008-0.06$ \\
\hline b-lactamase + & & & 2 & $0.12-4$ & & & 0.12 & $0.03-0.25$ \\
\hline \multicolumn{9}{|l|}{ Ciprofloxacin } \\
\hline All isolates & $0(0.0)$ & $0(0.0)$ & 0.015 & $0.008-1$ & N/A & N/A & 0.03 & $0.015-0.25$ \\
\hline b-lactamase + & $0(0.0)$ & $0(0.0)$ & 0.015 & $0.008-0.03$ & & & 0.03 & $0.015-0.25$ \\
\hline \multicolumn{9}{|l|}{ Levofloxacin } \\
\hline All isolates & $0(0.0)$ & $0(0.0)$ & 0.015 & $0.008-0.5$ & $0(0.0)$ & $0(0.0)$ & 0.03 & $0.015-0.25$ \\
\hline b-lactamase - & $0(0.0)$ & $0(0.0)$ & 0.015 & $0.008-0.5$ & $0(0.0)$ & $0(0.0)$ & $-a^{a}$ & $0.03-0.06$ \\
\hline b-lactamase + & $0(0.0)$ & $0(0.0)$ & 0.015 & $0.008-0.03$ & $0(0.0)$ & $0(0.0)$ & 0.03 & $0.015-0.25$ \\
\hline \multicolumn{9}{|l|}{ Moxifloxacin } \\
\hline All isolates & $0(0.0)$ & $0(0.0)$ & 0.03 & $0.008-0.25$ & $0(0.0)$ & $0(0.0)$ & 0.06 & $0.015-0.06$ \\
\hline b-lactamase - & $0(0.0)$ & $0(0.0)$ & 0.03 & $0.008-0.25$ & $0(0.0)$ & $0(0.0)$ & $-a^{a}$ & 0.06 \\
\hline b-lactamase + & $0(0.0)$ & $0(0.0)$ & 0.03 & $0.008-0.03$ & $0(0.0)$ & $0(0.0)$ & 0.06 & $0.015-0.06$ \\
\hline \multicolumn{9}{|l|}{ Tetracycline } \\
\hline All isolates & $5(1.0)$ & $14(2.7)$ & 1 & $0.12-16$ & $0(0.0)$ & $0(0.0)$ & 0.5 & $0.25-0.5$ \\
\hline b-lactamase - & $3(0.7)$ & $3(0.7)$ & 0.5 & $0.12-8$ & $0(0.0)$ & $0(0.0)$ & $-a^{a}$ & 0.25 \\
\hline b-lactamase + & $2(2.3)$ & $11(12.5)$ & 8 & $0.25-16$ & $0(0.0)$ & $0(0.0)$ & 0.5 & $0.25-0.5$ \\
\hline \multicolumn{9}{|l|}{ Co-trimoxazole } \\
\hline All isolates & $23(4.4)$ & $181(34.8)$ & 8 & $0.03-32$ & $6(4.3)$ & $2(1.4)$ & 0.5 & $0.06-4$ \\
\hline b-lactamase - & $21(4.9)$ & $130(30.1)$ & 8 & $0.03-32$ & $0(0.0)$ & $0(0.0)$ & $-\mathrm{a}$ & 0.12 \\
\hline b-lactamase + & $2(2.3)$ & $51(58.0)$ & 16 & $0.03-16$ & $6(4.3)$ & $2(1.5)$ & 0.5 & $0.06-4$ \\
\hline b-lactamase + & $1(1.1)$ & $10(11.4)$ & 8 & $0.12-16$ & $0(0.0)$ & $0(0.0)$ & 0.5 & $0.25-1$ \\
\hline
\end{tabular}

* Based on NCCLS breakp

a Not applicable, only 2 isolates. 
Table 6. PROTEKT 1999-2000: Distribution of macrolide resistance mechanisms among erythromycin-resistant (MIC $\geq 1 \mathrm{mg} / \mathrm{L}$ ) S. pyogenes isolated from Latin America in 1999-2000

\begin{tabular}{|c|c|c|c|c|c|c|}
\hline Center & $\begin{array}{c}\text { Total no. } \\
\text { of isolates tested }\end{array}$ & $\operatorname{erm}(\mathbf{B})$ & ermTR & mefA & $\operatorname{erm}(B)+m e f A$ & Total \\
\hline Argentina & 33 & & & & & \\
\hline Buenos Aires Center 50 & 23 & & & 3 & & 3 \\
\hline Buenos Aires Center 51 & 10 & & & 1 & & 1 \\
\hline Brazil & 145 & & & & & \\
\hline Florianópolis Center 41 & 24 & 1 & & 2 & & 3 \\
\hline Brasilia Center 45 & 19 & & & 2 & & 2 \\
\hline São Paulo Center 40 & 27 & & & & & 0 \\
\hline São Paulo Center 42 & 37 & & 2 & 2 & & 4 \\
\hline São Paulo Center 43 & 6 & & & & & 0 \\
\hline São Paulo Center 44 & 21 & & & & & 0 \\
\hline São Paulo Center 46 & 11 & & & & & 0 \\
\hline Mexico & 99 & & & & & \\
\hline Mexico City Center 31 & 58 & & 3 & 5 & & 8 \\
\hline Mexico City Center 32 & 10 & & & 2 & & 2 \\
\hline Monterrey Center 33 & 29 & & & 1 & 0 & 1 \\
\hline Latin America total & 277 & 1 & 5 & 18 & $\mathbf{0}$ & 24 \\
\hline
\end{tabular}

America, 2\% of S. pneumoniae isolates from Brazil were fully resistant to penicillin and $21.3 \%$ were intermediate; corresponding figures for Mexico were $20.8 \%$ and $28.6 \%$, respectively [13]. Finally, in the SENTRY programme, Sader et al. [4] analysed 344 community-acquired RTI pathogens isolated from Brazilian hospitals in 1997-1998. Among these, 2.3\% of the 176 pneumococcal isolates had full resistance to penicillin and $26.2 \%$ were intermediate. Considering all of these results together, the current PROTEKT findings suggest that nonsusceptibility to penicillin among pneumococci is increasing in Latin American countries. Furthermore, penicillin resistance appears to be shifting from intermediate towards full resistance in this region.

In addition to the increasing prevalence of penicillin resistance, we found considerable variation in resistance rates between centers within Brazil and Mexico. In Brazil, penicillin resistance ranged from $<5 \%$ in three centers to $30.0 \%$ in Brasilia. Differences were even more marked in Mexico, where rates ranged from
19.2\% in Monterrey to $76.5 \%$ in Mexico City. These differences may be explained because of the type of hospital involved, paediatric versus adult centers and primary or secondary care centers versus referral centers. Large inter-center variation in susceptibility has also been reported in other surveillance studies conducted in Latin America, including the LASER study [2], the Alexander Project [3], and the SENTRY programme [4]. In addition to inter-center variation, we detected a difference in susceptibility results attributable to methodology: two pneumococcal strains from center 41 (Florianópolis - Brazil) were reported as intermediate susceptibility to penicillin by E-test methodology when evaluated in the original center; however, when tested by the NCCLS broth microdilution method in the coordinating centre, both strains were classified as fully resistant towards penicillin. These findings re-affirm how important it is for physicians to be aware of local resistance patterns in order to ensure judicious use of antibacterials for 
patients with community-acquired RTIs that may be attributable to $S$. pneumoniae.

Another important finding of our study is the high prevalence of macrolide (erythromycin) resistance amongst pneumococci in Latin America. Overall, 15.3\% of the isolates were resistant to erythromycin, including $10.9 \%$ in Argentina, 6.5\% in Brazil and 27.6\% in Mexico. These values are comparable to the overall level of approximately $12 \%$ to $13 \%$ observed during the LASER study [2], the Alexander Project [3], and the SENTRY programme [4] and suggest that macrolide resistance is relatively stable in Latin America. However, as with penicillin resistance, marked intra-city/state variability was observed. Overall, macrolide resistance tended to be lower than penicillin resistance in most of the centers participating in the study, with the exception of the following: Florianópolis (Brazil), Monterrey and Mexico City Center 31.

We found the prevalence of macrolide resistance among $S$. pneumoniae to be high among isolates that were also resistant to penicillin (22.8\%). However, despite this cross-resistance between the macrolides and other MLS antibiotics, the ketolide telithromycin remained highly active against pneumococci, irrespective of concomitant penicillin and/or erythromycin resistance. Indeed, telithromycin was the most potent of the oral agents tested against $S$. pneumoniae $\left(\mathrm{MIC}_{90}=0.06 \mathrm{mg}\right.$ / $\mathrm{L})$. While fluoroquinolone resistance was absent in the Latin American centers participating in the SENTRY programme [4], the current PROTEKT findings indicate that the prevalence of $S$. pneumoniae resistance to fluoroquinolones may be increasing $(0.8 \%)$ and multipleresistance between the fluoroquinolones, $\beta$-lactams and a number of MLS antibacterial agents is apparent. This situation is of concern from the public health perspective and should be monitored carefully, particularly with the introduction of newer broad-spectrum fluoroquinolones for community-acquired RTIs.

Considering the other RTI pathogens monitored during the study, $16.9 \%$ of $H$. influenzae isolates in Latin America were â-lactamase positive, ranging from $11.0 \%$ in Brazil to $24.6 \%$ in Mexico. With the exception of ampicillin, amoxycillin and co-trimoxazole, resistance against the antibacterials tested in Latin America was not observed, although a high multiple-resistance to co-trimoxazole
(34.8\%) was detected. These rates of $\beta$-lactamase production and resistance patterns appear to be in agreement with those reported elsewhere $[4,14,3]$. In the SENTRY programmeconducted in the same region during 1997-1998 [4], 12.7\% of $361 \mathrm{H}$. influenzae isolates were found to be $\beta$-lactamase producers. Among the participating countries, rates of resistance were highest in Mexico (26\%), followed by Argentina (17.1\%), Chile (12.5\%), and Brazil (9.3\%). As in the PROTEKT study, a high rate of resistance to co-trimoxazole (40\%) was also observed. Also during 1997-1998, 9.4\% of the 223 Brazilian-isolates strains analysed by MRL were resistant to ampicillin and $2.2 \%$ were intermediate [14]. Resistance to co-trimoxazole (47.1\%) was high in this study. Finally, in the Alexander Project, $\beta$-lactamase production was detected in $10.3 \%$ of Brazilian isolates [3]. Infrequent resistance to chloramphenicol (11.9\%), along with a high frequency of resistance to co-trimoxazole $(29.1 \%)$, was observed in this study.

These findings suggest that susceptibility to the macrolides, the ketolide telithromycin and the fluoroquinolones is universally high in Latin America at present. However, the relatively high level of intermediate susceptibility to clarithromycin observed in the PROTEKT study needs to be monitored carefully as it may indicate the emergence of resistance to this agent among $H$. influenzae in Latin America.

$\beta$-lactamase production was highly prevalent amongst $M$. catarrhalis in Latin America, affecting 97.5\% of the isolates from Argentina, 98.6\% from Brazil, and $100 \%$ of isolates from Mexico $(98.6 \%$ overall). In comparison, data from the Alexander Project showed a rate of $90.4 \%$ in 1996 [3]; the corresponding rate of production in the SENTRY study in Latin America during 1997-1998 was 91.8\% [4]. With the exception of ampicillin and amoxycillin $(82.9 \%$ and $87.1 \%$ resistant isolates, respectively), $M$. catarrhalis was susceptible to the other antibacterial agents tested during the PROTEKT study. However, the apparent trend towards increasing $\beta$-lactamase production and the decreasing susceptibility of other â-lactams and also co-trimoxazole among $M$. catarrhalis in Latin America should be monitored carefully in the future. 
As anticipated, all Latin American S. pyogenes isolates remained susceptible to penicillin and most of the other $\beta$-lactams, with the exception of cefixime. In patients allergic to penicillins and other $\beta$-lactams, macrolides are useful agents for treating infections with this pathogen. However, recent reports have found that, in some countries, up to $30 \%$ of $S$. pyogenes isolates are resistant to macrolides $[16,17]$. In PROTEKT, the prevalence of macrolide resistance among $S$. pyogenes was found to be $8.3 \%$. Telithromycin was active against $100 \%$ of Latin American S. pyogenes isolates, suggesting that it is a useful alternative to macrolides in the treatment of $S$. pyogenes infections in penicillinallergic patients.

The PROTEKT findings also showed that the prevalence of methicillin resistance among isolates of S. aureus (MRSA, 26.5\%) is similar to that reported by the Latin American centers participating in the SENTRY surveillance programme between 1997 and $1999(34.9 \%)$ [18].

In summary, the current findings from the Latin American centers participating in the PROTEKT study confirm that antibacterial resistance among common community-acquired RTI pathogens is an emerging problem in Latin America. The results suggest that the already high levels of pneumococcal resistance to the $\beta$-lactam and macrolides reported from other large surveillance studies conducted in this region are now being exceeded. In particular, resistance to the $\beta$ lactams appears to be increasing and fluoroquinolone resistance in $S$. pneumoniae has been detected, albeit at a low level. These findings highlight the need for increased vigilance in monitoring and tracking changes in antibacterial susceptibility in order to guide empirical therapy. With this in mind, findings from the ongoing PROTEKT study are rapidly available and frequently updated on the World Wide Web (www.protekt.org), thereby providing up-to-date susceptibility data for physicians to facilitate local prescribing decisions. Such steps, along with the development of new agents that do not induce or create conditions for selection for resistance, for example the novel ketolide telithromycin, are vitally important in order to safeguard future antibacterial efficacy.

\section{Acknowledgements}

The PROTEKT study was funded by Aventis. Microbiological analysis was carried out by GR Micro Ltd, London, UK and data analysis was carried out by Micron Research Ltd, Upwell, Cambs, UK.

\section{References}

1. Odland B.A., Jones R.N., Verhoef J., et al. Antimicrobial activity of gatifloxacin (AM-1155, CG5501), and four other fluoroquinolones tested against 2,284 recent clinical strains of Streptococcus pneumoniae from Europe, Latin America, Canada, and the United States. The SENTRY Antimicrobial Surveillance Group (Americas and Europe). Diagn Microbiol Infect Dis 1999;34:315-20.

2. Jacobs M.R., Appelbaum P.C. Susceptibility of 1100 Streptococcus pneumoniae strains isolated in 1997 from seven Latin American and Caribbean countries. Laser Study Group. Int J Antimicrob Agents 2000;16:17-24.

3. Felmingham D., Washington J. Trends in the antimicrobial susceptibility of bacterial respiratory tract pathogens findings of the Alexander Project 1992-1996. J Chemother 1999; 11(Suppl. 1):5-21.

4. Sader H.S., Gales A.C., Granacher T.D., et al. Prevalence of antimicrobial resistance among respiratory tract isolates in Latin America: results from SENTRY antimicrobial surveillance program (1997-1998). Braz J Infect Dis 2000;4:245-54.

5. Felmingham D., Zhanel G., Hoban D. Activity of the ketolide antibacterial telithromycin against typical community-acquired respiratory pathogens. J Antimicrob Chemother 2001;48(Suppl. T1):33-42.

6. Balfour J.A., Figgitt D.P. Telithromycin. Drugs 2001;61:815-29.

7. Felmingham D., Grüneberg R.N. A multicentre collaborative study of the antimicrobial susceptibility of community-acquired, lower respiratory tract pathogens 1992-1993: the Alexander Project. J Antimicrob Chemother 1996;38(Suppl. A):1-57.

8. Grüneberg R.N., Felmingham D. Results of the Alexander Project: a continuing multicenter study of the antimicrobial susceptibility of community-acquired lower respiratory tract bacterial pathogens. Diagn Microbiol Infect Dis 1996;25:169-81.

9. National Committee for Clinical Laboratory Standards. Methods for dilution antimicrobial susceptibility tests for bacteria that grow aerobically. Approved standard - fifth edition. NCCLS document M100-S12, 2002, NCCLS, Wayne, PA, 19087. 
10. National Committee for Clinical Laboratory Standards. Performance standards for antimicrobial susceptibility testing; Twelfth Informational Supplement. NCCLS document M2-A7 and M7-A5, 2002, NCCLS, Wayne, PA, 19087.

11. Farrell D.J., Morrissey I., Bakker S., Felmingham D. Detection of macrolide resistance mechanisms in Streptococcus pneumoniae and Streptococcus pyogenes using a multiplex rapid cycle PCR with microwell-format probe hybridization. J Antimicrob Chemother 2001; 48:541-4.

12. Sader H.S., Jones R.N., Ballow C.H., et al. Antimicrobial susceptibility of quinupristin/dalfopristin tested against gram-positive cocci from Latin America: results from the global SMART (GSMART) surveillance study. Braz J Infect Dis 2001;5:21-31.

13. Hortal M., Ruvinsky R., Rossi A. et al. [Impact of Streptococcus pneumoniae on pneumonia in Latin American children. SIREVA-Vigia Group.] Rev Panam Salud Publica 2000;8:185-95.

14. Critchley I.A., Hickey M.L., Barth A.L., et al. Antimicrobial resistance surveillance of 1997-1998 isolates of Streptococcus pneumoniae, Haemophilus influenzae, and Moraxella catarrhalis from Brazil (abstract A89). $99^{\text {th }}$ General Meeting of the American Society for Microbiology; Chicago, IL, 1999.

15. Cocuzza C.E., Tomasini A., Renzetti D., et al. Ketolide (HMR 3647) in vitro activity on 4000 strains of S. pyogenes isolated in Northern Italy (abstract 1.06). 4th International Conference on the Macrolides, Azalides, Streptogramins and Ketolides; Barcelona, Spain 1998. Available from: URL:http://www.icmask.org/icmask 04/icmask04 posters/ section 01/abs0106.html. Accessed October 02, 2002.

16. Hoban D., Palatnick L., Weshnoweski B., et al. The novel ketolide HMR 3647 is highly active against worldwide isolates of Streptococcus pneumonia and Streptococcus pyogenes (abstract 1.04). 4th International Conference on the Macrolides, Azalides, Streptogramins and Ketolides 1998; Barcelona, Spain. Available from: URL:http:// www.icmask.org/icmask 04/icmask04 posters/ section 01/abs0104.html. Accessed October 02, 2002.

17. Appelbaum P.C., Kelly L.M., Hryniewicz W., et al. Activity of telithromycin against $300 \mathrm{~S}$. pyogenes from ten central and eastern European countries (abstract 2153). In: Abstracts: 40th Interscience Conference on Antimicrobial Agents and Chemotherapy, Toronto, Canada, 2001.

18. Diekema D.J., Pfaller M.A., Schmitz F.J., et al. and the SENTRY Participants Group. Survey of infections due to Staphylococcus species: frequency of occurrence and antimicrobial susceptibility of isolates collected in the United States, Canada, Latin America, Europe, and the Western Pacific region for the SENTRY Antimicrobial Surveillance Program, 1997-1999. Clin Infect Dis 2001;32(Suppl. 2):S114-S32. 